\title{
Glomus tumors in neurofibromatosis type 1: genetic, functional and clinical evidence of a novel association
}

\author{
Hilde Brems ${ }^{\star}, 1$, Caroline Park ${ }^{*}, 2$, Ophélia Maertens ${ }^{3}$, Alexander Pemov ${ }^{4}$, Ludwine \\ Messiaen $^{5}$, Meena Upadhyaya ${ }^{6}$, Kathleen Claes ${ }^{7}$, Eline Beert ${ }^{1}$, Kristel Peeters ${ }^{1}$, Victor \\ Mautner ${ }^{8}$, Jennifer L. Sloan ${ }^{9}$, Lawrence Yao ${ }^{10}$, Chyi-Chia Richard Lee ${ }^{11}$, Raf Sciot ${ }^{12}$, Luc \\ De Smet ${ }^{13}$, Eric Legius ${ }^{1}$, and Douglas R. Stewart ${ }^{4}$ \\ ${ }^{1}$ Department of Human Genetics, Catholic University Leuven, 3000 Leuven, Belgium \\ ${ }^{2}$ Albert Einstein College of Medicine, Bronx, New York, 10461 USA \\ ${ }^{3}$ Genetics Division, Department of Medicine, Brigham and Women's Hospital and Harvard Medical \\ School, Boston, Massachusetts 02115 USA \\ ${ }^{4}$ Genetic Disease Research Branch, National Human Genome Research Institute, National \\ Institutes of Health, Bethesda, Maryland 20892 USA \\ ${ }^{5}$ Department of Genetics, Medical Genomics Laboratory, University of Alabama at Birmingham, \\ Birmingham, Alabama 35294 USA \\ ${ }^{6}$ Institute of Medical Genetics, Cardiff University, Heath Park, Cardiff CF14 4XN, UK \\ ${ }^{7}$ Center for Medical Genetics, Ghent University Hospital, 9000 Gent, Belgium \\ ${ }^{8}$ Laboratory for Tumor Biology and Developmental Disorders, Department of Maxillofacial Surgery, \\ University Hospital Eppendorf, Martinistrasse 52, 20246 Hamburg, Germany \\ ${ }^{9}$ Genetic and Molecular Biology Branch, National Human Genome Research Institute, National \\ Institutes of Health, Bethesda, Maryland 20892 USA \\ ${ }^{10}$ Department of Radiology, Clinical Center, National Institutes of Health, Bethesda, Maryland 20892 \\ USA \\ ${ }^{11}$ Laboratory of Pathology, National Cancer Institute, National Institutes of Health, Bethesda, \\ Maryland 20892 USA \\ ${ }^{12}$ Department of Pathology, Catholic University Leuven, 3000 Leuven, Belgium \\ ${ }^{13}$ Department of Orthopaedic Surgery, University Hospital Pellenberg, 3212 Lubbeek, Belgium
}

\section{Abstract}

\begin{abstract}
Neurofibromatosis type 1 (NF1) is a common disorder that arises secondary to mutations in the tumor suppressor gene NF1. Glomus tumors are small, benign but painful tumors that originate from the glomus body, a thermoregulatory shunt concentrated in the fingers and toes. We report eleven individuals with NF1 who harbored 20 glomus tumors of the fingers and one in the toe; five individuals had multiple glomus tumors. We hypothesized that bi-allelic inactivation of $N F 1$ underlies the pathogenesis of these tumors. In twelve NF1-associated glomus tumors, we used cell
\end{abstract}

\footnotetext{
Requests for reprints Douglas R. Stewart, National Human Genome Research Institute, 49 Convent Drive Bldg 49, Rm 4A62, Bethesda, MD 20892. drstewart@mail.nih.gov..

These authors contributed equally

Conflict of Interest The authors report no relevant conflicts of interest.
} 
culture and laser capture micro-dissection to isolate DNA. We also analyzed two sporadic (not-NF1associated) glomus tumors. Genetic analysis showed germline and somatic $N F 1$ mutations in seven tumors. RAS-MAPK hyper-activation was observed in cultured $\mathrm{NF}^{-/-}$glomus cells, reflecting a lack of inhibition of the pathway by functional neurofibromin, the protein product of $N F 1$. No abnormalities in NF1 or RAS-MAPK activation were found in sporadic glomus tumors. By comparative genomic hybridization, we observed amplification of the 3 '-end of CRTACl and a deletion of the 5'-end of WASF1 in two NF1-associated glomus tumors. For the first time, we show that loss of neurofibromin function is crucial in the pathogenesis of glomus tumors in NF1. Glomus tumors of the fingers or toes should be considered as part of the tumor spectrum of NF1.

\section{Keywords}

Neurofibromatosis type 1; Glomus tumor; Comparative genomic hybridization; RAS-MAPK hyperactivation; Bi-allelic inactivation

\section{Introduction}

Neurofibromatosis type 1 (NF1) is a common (1/3000), autosomal dominant disorder that arises secondary to mutations in the tumor suppressor gene $N F 1$ (1). The protein product of NF1, neurofibromin, regulates RAS through its GTPase activating protein (GAP)-related domain (2). Individuals with NF1 are at an increased risk for a variety of benign and malignant tumors. Biallelic inactivation (a "second hit") (3) of NF1 due to loss of heterozygosity ( $\mathrm{LOH}$ ) or somatic mutation is pathogenic in a variety of NF1-associated tumors (4).

Glomus tumors are benign neoplasms that arise from the glomus body, a specialized thermoregulatory shunt concentrated in the fingers and toes (5). (Glomus tumors in the fingers or toes are distinct from adrenal and extra-adrenal paragangliomas, also called "glomus tumors" (6).) The glomus body is a highly innervated structure containing an afferent arteriole, an anastomotic Suquet-Hoyer canal and an efferent venule. The canal is surrounded by concentric layers of contractile alpha smooth muscle actin ( $\alpha$ SMA)-positive glomus cells. Heat-induced contraction of the glomus body causes closure of the arteriovenous anastomosis and forces blood flow through the capillary network of the distal phalanx, causing heat loss (7). Cold temperatures prompt relaxation of the glomus body, opening the anastomosis and conserving body heat.

Sporadic glomus tumors of the fingers are solitary and predominantly affect middle-aged women $(5,8,9)$. Affected individuals present with a triad of severe paroxysmal pain, cold intolerance and localized tenderness. The first association of NF1 and glomus tumors (at any location) was published in 1938 (10). To date, there are eight published cases of an NF1 association and glomus tumors of the fingers or toes in the English-language literature (11-15). There were no examples of multi-focal glomus tumors in two large retrospective reviews of 86 sporadic cases $(8,9)$. However, of the eight individuals with NF1 and glomus tumors of the fingers or toes, seven harbored multiple tumors $(11,12,14,15)$, suggesting an association.

We hypothesized that bi-allelic inactivation of $N F 1$ is pathogenic in NF1-associated glomus tumors (14). In this report, we searched for somatic NF1 mutations, loss of neurofibromin function, and dysregulation of the RAS-MAPK pathway in glomus cells in NF1-associated and sporadic glomus tumors. We also investigated genome copy number changes using comparative genomic hybridization. 


\section{Materials and Methods}

\section{Patient material}

Studies were performed on 21 glomus tumors from eleven individuals with NF1. Fresh tissue was available from nine tumors and was used for primary cell cultures. The three glomus tumors from NF1-G10 were fixed in Histochoice (an alcohol-based fixative, Sigma, St. Louis, MO) prior to laser capture micro-dissection (LCM). Primary glomus cell cultures were also established from two tumors from two individuals without NF1. Skin fibroblast culture from a normal control was also available.

\section{Cell culture}

Surgically excised glomus tumors were treated overnight with collagenase $(160 \mathrm{U} / \mathrm{ml})$ and dispase $(0.8 \mathrm{U} / \mathrm{ml})$ at $37^{\circ} \mathrm{C}$. Glomus cells were grown to confluency in Dulbecco's Modified Eagle Medium: Nutrient Mixture F-12 (DMEM/F12) + 10\% fetal bovine serum (FBS) + penicillin + streptomycin and harvested.

\section{Immunocytochemistry}

For immunofluorescent staining, cells cultured from glomus tumors were fixed with $4 \%$ paraformaldehyde, permeabilized with $0.1 \%$ Triton X-100 and blocked with $10 \%$ FBS. The cells were washed with phosphate buffered saline (PBS) with 1\% FBS and incubated with mouse anti- $\alpha$ SMA antibody (1:400; Sigma A2547, Sigma, Bornem, Belgium). Wash with PBS with $1 \%$ FBS was followed by incubation with a fluorescent goat anti-mouse antibody (Alexa Fluor 488, Invitrogen, Merelbeke, Belgium). Slides were mounted with mounting medium (Vectashield, Vector Laboratories, Peterborough, UK) and cell nuclei visualized with 4', 6diamidino-2-phenylindole (DAPI).

\section{DNA isolation from paraffin-embedded tissue}

Eight-micron sections from the three glomus tumors from patient NF1-G10 were mounted onto PEN membrane slides (Zeiss, Thornwood, NY, USA) and stained with hematoxylin and eosin. Lesional tumor cells were micro-dissected from surrounding stroma and vasculature with either the PALM II (Zeiss) or the PixCell II (Molecular Devices, Sunnyvale, CA, USA) LCM systems. The tissue fragments were digested in proteinase $\mathrm{K}$ and DNA extracted per manufacturer's instruction (Picopure DNA extraction kit, Molecular Devices). For single nucleotide polymorphism - comparative genomic hybridization (SNP-CGH), LCM material was re-suspended in buffer containing $0.5 \mathrm{M}$ TrisHCl ( $\mathrm{pH}$ 9.0), 0.5\% SDS and $5 \mathrm{mM}$ EDTA. Proteinase K (Invitrogen, Carlsbad, CA, USA) at $400 \mu \mathrm{g} / \mu \mathrm{L}$ was added, and samples were incubated at $55^{\circ} \mathrm{C}$ for 16 hours. Samples were extracted with phenol/chloroform and DNA was precipitated. The DNA concentration was quantified using Picogreen (Invitrogen) and/or by spectrophotometer (NanoDrop ND-1000, Thermo Scientific, Wilmington, DE, USA) analysis at $260 \mathrm{~nm}$.

\section{Whole genome amplification}

The DNA extracted from tumors \#1, \#2 and \#3 from patient NF1-G10 was subjected to whole genome amplification (WGA) by either multiple displacement amplification (MDA) with the Repli-G kit (Qiagen) or a PCR-based method with the GenomePlex kit (Sigma). The manufacturer's instructions were followed with appropriate controls.

\section{Germline and somatic NF1 mutation detection and loss of heterozygosity analysis}

Germline mutation screening of $N F 1$ was performed on cDNA from puromycin-treated lymphocytes (16). Detected mutations were confirmed on genomic DNA. NF1 somatic mutation analysis was performed using the same technique on cell cultures derived from 
glomus tumors. Somatic NF1 mutation analysis was performed on WGA DNA from tumors \#1, \#2 and \#3 subjected to LCM from participant NF1-G10 as per published methods (17, 18). Somatic mutations of $N F 1$ were confirmed on non-amplified tumor genomic DNA. Multiplex ligand-dependent probe amplification (MLPA) was used to assay exonic deletions (19). LOH analysis on DNA from cell cultures from glomus tumors (tumor \#1, NF1-G4; tumor \#1, NF1-G6; tumor \#1, NF1-G7; tumor \#1, NF1-G9) was performed by genotyping microsatellite markers telomeric to (3'NF1-3, 3'NF1-1) (20) and within NF1 (Alu, IVS27AC33.1, IVS38GT53.0, IVS27TG24.8) (21-24).

\section{Bi-allelic assignment of somatic and germline NF1 mutations}

Germline and somatic NF1 mutations may occur on the same or different alleles. In non-WGA tumor DNA from NF1-G10, SNPs rs2269855 and rs 7350946 were co-amplified by PCR with NF1 somatic mutations \#2 and \#3; in germline DNA from NF1-G10, SNP rs2525565 was coamplified with the $N F 1$ germline mutation (Table 1). The PCR products were sub-cloned into the TOPO-TA vector (Invitrogen), and transformed into DH5 $\alpha$ cells, harvested and sequenced. To create NF1 haplotypes, nine informative NF1 SNPs were genotyped in participant NF1G10 and family members (Figure 1). Assignment of status (wild-type or NF1 mutation) to the haplotypes was then determined by segregation analysis within the family.

\section{Bisulfite modification and HUMARA-MSP to assess clonality of glomus tumors}

We used the human androgen receptor assay - methylation-specific PCR (HUMARA-MSP) (25), with minor modifications (Supplemental Table 1) to determine the clonality of the three tumors from NF1-G10 (26). Bisulfite modification was performed using the Epitect bisulfite modification kit (Qiagen, Valencia, CA, USA) according to the manufacturer's instructions. Bisulfite-modified DNA was used in the HUMARA-MSP assay using Amplitaq Gold 2x PCR mastermix (Applied Biosystems, Foster City, CA, USA) in a thermal cycler (MJ Research, Waltham, MA, USA). PCR products were analyzed with an ABI 3100 Genetic Analyzer (Applied Biosystems) using GeneMapper software (version 3.1, Applied Biosystems). Germline DNA from NF1-G10 was used as a polyclonal control. As a monomorphic control, germline DNA from three females with oculofaciocardiodental syndrome was used (27).

\section{RAS-MAPK pathway analysis of glomus cells}

Cell culture of glomus tumors (NF1-associated glomus tumor-derived glomus cells, NF1associated glomus tumor-derived fibroblasts, sporadic (non-NF1) glomus tumor-derived glomus cells and control fibroblasts (skin fibroblasts from an individual not affected with NF1)) were grown to confluence, starved overnight in serum-free medium and stimulated with acidic fibroblast growth factor (aFGF, Sigma, Bornem, Belgium) $(10 \mathrm{ng} / \mathrm{mL})$ for 5, 15, 30, 45 and 60 minutes. Cell lysates were analyzed by Western blot. DNA was also extracted from the NF1-associated glomus tumor-derived glomus cells to confirm the somatic and germline NF1 mutations. Antibodies used for immunoblotting included anti-phospho-MEK1/2 (Cell Signaling Technology, Beverly, MA, USA), anti-MEK1/2 (Santa Cruz Biotechnology, Santa Cruz, CA, USA), anti-phospho-ERK1/2 (Cell Signaling), anti-ERK1/2 (Cell Signaling), and anti- $\beta$-actin (Sigma, Bornem, Belgium). Quantitative analysis of Western blot images was performed using Scion software (Scion Corp, Frederick, MD, USA). Experiments were performed in triplicate. Statistical significance was determined by multivariate repeated measures ANOVA.

\section{Comparative genomic hybridization}

Illumina HumanHap550 single nucleotide polymorphism comparative genomic hybridization (SNP-CGH)—Un-amplified DNA ( 500 ng) microdissected from both glomus tumors \#1 and \#3 and germline DNA from NF1-G10 was hybridized to Illumina 
HumanHap550 Genotyping BeadChips (Illumina, San Diego, CA, USA). Data was analyzed using Illumina BeadStudio software version 3.1 with genotyping module version 3.2.23. To assess LOH and copy-number changes, we used the "LOH Score" and "CNV partition" algorithms. The recommended thresholds for a significant $\mathrm{LOH}$ score is $>5$ and for the confidence score is 100 ("DNA Copy Number Analysis Algorithms," Illumina publication number 970-2007-008, March 12, 2008). Genomic coordinates for all SNPs were derived from dbSNP build 129.

We also identified discordant loci by comparing SNP genotypes of tumor and corresponding germline DNA at each SNP locus. For those SNPs with apparent LOH, we then identified the nearest centromeric and telomeric heterozygous (informative) SNPs to establish an interval with putative $\mathrm{LOH}$.

Agilent oligo array comparative genomic hybridization (Array-CGH)-Array-CGH using the Agilent Human Genome Microarray Kit 244A (Agilent Technologies, Diagem, Belgium) was performed on tumor DNA from primary glomus tumor cell cultures from participants NF1-G1 (tumor \#1), NF1-G3 (tumor \#3), NF1-G8 (tumor \#1), non-NF1-G1, and non-NF1-G2. The presence of a somatic NF1 mutation was confirmed in each culture of NF1associated glomus tumor-derived glomus cells tested by array-CGH. Matching genomic DNA was available for the three tumors from individuals with NF1. For the tumors from individuals not affected with NF1, we used gender-matched genomic DNA from a healthy control. Digestion, labeling and hybridization were performed according to the manufacturer's instructions ("Agilent Oligonucleotide Array-based CGH for Genomic DNA Analysis" protocol, v4.0, June 2006). Microarrays were scanned by the Genepix 4000B scanner (Axon Instruments, Molecular Devices, Sunnyvale, CA, USA) and analyzed by the Agilent Feature Extraction software (v9.5.1). Results were visualized by Agilent CGH Analytics software (v3.5.14).

\section{Quantitative PCR at the CRTAC1, GUCY1A2, and WASF1 loci}

Three loci (CRTAC1, GUCY1A2, WASF1) with SNP-CGH evidence of copy-number changes were evaluated with custom TaqMan PCR assays (Applied Biosystems) according to the manufacturer's instructions. Unamplified genomic DNA (1 ng) isolated by LCM from glomus tumors \#1 and \#3 and germline DNA (1 ng) were used in the qPCR reactions. All reactions were performed in triplicate in a 7900HT Fast Real-Time PCR instrument (Applied Biosystems). Relative amounts of DNA in each sample/locus were calculated using the standard "double delta" Ct method and expressed as percentage of the DNA in the germline sample. Primers are listed in Supplemental Table 1. RNase P RNA component H (RPPHI) was used as a normalizing control. For each gene, a standard t-test was used to test for significance of differences in relative DNA amount between germline and tumor samples.

\section{Results}

\section{Clinical characterization of NF1-associated glomus tumors}

We evaluated eleven individuals (three males and eight females, ages: 11-59 years; mean age excluding the child: 40 years) with signs and symptoms of NF1 with 21 pathologically confirmed glomus tumors of the fingers and toes (Table 1). One individual (NF1-G6) was diagnosed with mosaic NF1 (previously reported patient SNF1-1) (28). The remaining ten individuals fulfilled the consensus criteria for the diagnosis of NF1. The clinical data of two of these ten individuals have been previously reported (NF1-G2 as "Case 2" and NF1-G3 as "Case 1") (14). A pathogenic germline mutation in NF1 was identified in all individuals, with the exception of N1-G6 (mosaic NF1), for whom the mosaic NF1 mutation was found in neurofibroma-derived Schwann cells (28). 
Five of the eleven individuals harbored multiple glomus tumors. Twenty of the 21 glomus tumors were located in the fingertips, with the exception of NF1-G9, whose tumor was located in the right hallux. Ten tumors were located in the right hand, and ten in the left hand. The aggregate distribution of tumors was similar for both hands: F1/thumb (5\%), F2 (5\%), F3 $(25 \%)$, F4 (50\%), F5 (15\%).

\section{NF1-associated glomus tumors demonstrate typical glomus tumor morphology}

Histological examination of all 21 tumors revealed small vessels surrounded by uniform cuboidal cells without cytologic atypia, necrosis or increased mitotic activity (Figure 2A). Immunostaining with $\alpha$ SMA showed uniformly positive cytoplasmic staining in the tumor cells (Figure 2B).

\section{NF1-associated glomus tumors arise secondary to somatic NF1 mutations in alpha smooth muscle actin - positive glomus cells}

We used two strategies to collect DNA from small glomus tumors: 1) fresh tissue culture to derive glomus cells and fibroblasts (nine tumors) and, 2) LCM of paraffin-embedded tissue (three tumors).

In the cultured tumors, the glomus cells were $\alpha$ SMA positive (Figure $2 \mathrm{~B}$ ). In addition to the germline mutations, five somatic mutations were identified in five of the nine tumors (Table 1). No NF1 mutations were detected in glomus cells or fibroblasts from two sporadic glomus tumors. In the three tumors subject to LCM, two different somatic mutations were identified in two tumors (Table 1) in addition to the germline mutation. All seven somatic mutations are predicted to inactivate $N F 1$. Using segregation analysis of $N F 1$ SNP haplotypes harboring the NF1 germline and somatic mutations in the pedigree of NF1-G10, we proved that the germline and somatic mutations arose on separate copies of chomosome 17 (Figure 1).

\section{NF1-associated glomus tumors are monoclonal by HUMARA-MSP}

Since NF1-associated glomus tumors arise from bi-allelic inactivation of $N F 1$, we reasoned that they should harbor evidence of a monoclonal expansion from a single cell. In three different glomus tumors from three fingers of one female (NF1-G10), a single allele was detected using the methylated- and unmethylated-specific primer pairs (Supplemental Figure 1), consistent with monoclonal tumor expansion.

\section{Bi-allelic inactivation of NF1 in NF1-associated tumor-derived glomus cells increased activation of the RAS-MAPK pathway compared to other NF1-associated cells and control skin fibroblasts}

Since bi-allelic inactivation of $N F 1$ was observed in NF1-associated tumor-derived glomus ( $\alpha$ SMA-positive) cells, we reasoned that MAPK pathway activity should be elevated when compared to NF1-associated glomus tumor-derived ( $\alpha$ SMA-negative) fibroblasts, sporadic glomus tumor-derived glomus ( $\alpha$ SMA-positive) cells and control skin fibroblasts.

Consistent with the predicted effects of NF1 bi-allelic inactivation, we observed significantly higher MAP-kinase kinase 1 and 2 (MEK1/2) phosphorylation ratios in NF1-associated glomus tumor-derived glomus cells when compared with the three cell types described above at 5,15 and 30 minutes after stimulation with acidic fibroblast growth factor (aFGF) (Figure 3). The NF1-associated glomus tumor-derived fibroblasts showed less MEK1/2 activation in comparison to the NF1-associated glomus tumor-derived glomus cells but a higher activation at 5 and 15 minutes when compared to sporadic glomus tumor-derived glomus cells and control skin fibroblasts. 
Similarly, we observed increased activation of extracellular signal-regulated kinase 1 and 2 (ERK1/2) phosphorylation after stimulation with aFGF (Figure 3). The maximum pERK/ERK ratio was detected in the NF1-associated glomus tumor-derived glomus cells 15 minutes after stimulation; the pERK/ERK ratios did not return to pre-stimulation levels even 60 minutes after stimulation. The NF1-associated glomus tumor-derived fibroblasts, sporadic glomus tumor-derived glomus cells and skin fibroblasts showed a similar but significantly lower increase in pERK/ERK ratios after stimulation with aFGF at all time points; 30 minutes after stimulation, the pERK/ERK ratio in these three cell types returned to pre-stimulation levels. Taken together, these data are consistent with the effects of $N F 1$ bi-allelic inactivation on the MAPK pathway in NF1-associated glomus tumor-derived glomus cells $(29,30)$.

\section{Copy number changes at the CRTAC1 and WASF1 loci}

\section{Illumina Human Hap550 SNP comparative genomic hybridization and quantitative PCR with micro-dissected tumor DNA-Supplemental Table 2 lists loci} with evidence of copy number alterations in glomus tumors \#1 and \#3 (from NF1-G10), but not in germline DNA. All CNVs detected were located at least $20 \mathrm{~kb}$ (often substantially more) from a known gene, except for four that were found to be within CRTACl (glomus tumors \#1 and \#3), WDR78, GUCY1A2 and VPS13C (glomus tumor \#3 only).

Since the LOH score and CNV partition score are insensitive to the detection of copy number changes at a single SNP, we sought discrepancies in SNP genotypes between tumor and germline samples. One SNP (rs4945851, intron 1 of WASF1) was discrepant (Figure 4). There was no evidence of a copy number change at the two closest flanking SNPs (rs6568634 and rs7761436), thus delimiting the size of the WASF1 putative deletion to $\sim 10 \mathrm{~kb}$.

Due to severe limitations of the availability of tumor DNA, we performed qPCR on CRTACI, $W A S F 1$ and GUCY1A2. In both CRTAC1 and WASF1, we observed copy-number changes consistent with those observed in the SNP-CGH data (Figure 5). We were unable to confirm the homozygous deletion in tumor \#3 of the GUCY1A2 locus.

Agilent oligonucleotide array comparative genome hybridization with DNA from cell culture-Oligonucleotide array-CGH analysis did not show copy number changes in the cultured cells from the glomus tumors with proven $N F 1$ inactivation (data not shown), more specifically, the loci identified by the Illumina platform on DNA extracted from paraffinembedded tissues were normal. Similarly, the two glomus tumors from individuals not affected with NF1 and the non-NF1 control fibroblasts did not show any copy number alterations.

\section{Discussion}

In this report, we present the first genetic and molecular proof of an association of glomus tumors of the fingers and toes with NF1. The mean age of adult participants in our study (40 years) is comparable to that of the sporadic glomus tumor population (9). However, one participant in our study (NF1-G7) was only 11 years old. We also observed multiple glomus tumors in $45 \%$ of our participants, a feature not observed in sporadic glomus tumors. In tumor predisposition syndromes like NF1, an early age of onset and the presence of multi-focal tumors are evidence of an association.

Glomus tumors are small (typically $<5 \mathrm{~mm}$ ). We used two techniques to obtain tumor DNA. We identified both germline and somatic mutations in $N F 1$ in six tumors; in a seventh tumor we found an $N F 1$ germline mutation plus LOH $(1 / 7=14 \%$; $95 \%$ confidence interval: 0.003 0.578). The rate of $\mathrm{LOH}$ we detected in glomus tumors is not significantly different from the expected 25\% observed in neurofibromas (binomial distribution) (31) due to our modest sample size. In two tumors we showed that the wild-type chromosome harbored the somatic mutation 
(the "second hit" of Knudson's two-hit hypothesis). Bi-allelic inactivation of $\mathrm{NFl}$ is a common pathogenic mechanism of NF1-associated tumors. In four tumors from two individuals, four different somatic NF1 mutations were identified, suggesting that the multi-focal NF1associated glomus tumors arise from independent events. Presumably NF1-nullizygosity arises in glomus cells secondary to mitotic DNA replication errors in NF1; this matters since glomus cells rely on neurofibromin-dependent RAS-MAPK-related growth factor cascades.

We also sought evidence of the functional consequences of the inactivation of NF1. Biallelic inactivation of NF1 in NF1-associated glomus tumor-derived glomus cells results in an increased activation of the MAPK pathway, as observed in other tumor cells with bi-allelic inactivation of $N F 1(29,30)$. Biochemical analysis of NF1-associated tumor-derived glomus cells (with germline and somatic NF1 mutations) showed stronger and longer activation of the MAPK pathway after stimulation with aFGF when compared to NF1-associated tumor derived fibroblasts (with a germline NFI mutation only), sporadic tumor derived glomus cells (no NF1 mutations) and normal skin fibroblasts (no NF1 mutations). In three (of three) glomus tumors from three different fingertips from a single female, $\mathrm{X}$-inactivation as detected by the HUMARA-MSP assay was consistent with monoclonal expansion of the glomus tumors. Such an expansion is compatible with the consequences of bi-allelic inactivation of a tumor suppressor gene (such as NF1) in a single cell (32).

We performed a genome-wide search for copy number changes in both cultured and paraffinembedded tumor cells. In participant NF1-G10 tumor \#1 (no somatic NF1 mutation identified) and tumor \#3 (bi-allelic NF1 inactivation), qPCR was consistent with a partial deletion of a portion of the 5'-untranslated region of WASF 1 (Figure 5). Interestingly, WASF1 forms a bidirectional gene pair with the $5^{\prime} C D C 40$. The bi-directional promoter of WASF1 and CDC4O is located within the putative deletions of both tumors from NF1-G10. Deletion of the bidirectional promoter may plausibly affect expression of both $W A S F 1$ and $C D C 40$. There is significant over-representation of bidirectional promoters associated with cancer-related genes (33); their role in benign tumors is unknown. WASF1 is down-regulated in ovarian cancer (34). There are no reported mutations in human $C D C 40$, an orthologue of yeast $C D C 40$, which is a controller of cell cycle arrest (35). Both WASF1 and CDC40 are candidates for further investigation in glomus tumors.

A $\sim 50 \mathrm{~kb}$ amplification within CRTACl was also observed in both glomus tumors from NF1G10. CRTACl encodes human cartilage acidic protein 1 and is useful in distinguishing chondrocyte-like, osteoblast-like and mesenchymal stem cells in culture (36). The PRINTS database $^{1}$ predicts a C-terminal antifreeze type I domain in CRTACl. Antifreeze proteins (AFPs) were identified in polar fish as an adaptation to survive in hypothermic conditions preventing cell damage (37). In prolonged sub-zero cryopreservation, AFPs protect the heart from freezing, improve survival and reduce apoptosis (38). Antifreeze domains are rare in the human genome. Their role in the pathogenesis of glomus tumors, derived from cold-responsive contractile glomus cells, is unknown.

Analysis of the cultured NF1 tumor derived glomus cells by array-CGH did not show copy number alterations. It is possible that the cell culture procedure selected for glomus cells without copy number alterations.

Lastly, many neural crest-derived cell types are involved in NF1 (39). Three observations from our data support a neural crest origin for glomus cells and their cognate tumors. First, glomus cells are $\alpha$ SMA-positive; progenitor cells cultured from rat sciatic nerve suggest that neural

${ }^{1}$ The PRINTS Fingerprint Database [Internet]. The Bioinformatics Group, School of Biological Sciences, The University of Manchester. Version 39.0. [cited April 15, 2009]. Available from: http://www.bioinf.manchester.ac.uk/dbbrowser/PRINTS/index.php 
crest stem cells generate $\alpha$ SMA-positive myofibroblasts (40). Second, the five NF1-associated tumor-derived cell cultures with a somatic NF1 mutation showed that only aSMA-positive glomus cells, and not tumor-derived fibroblasts, harbored somatic and germline NF1 mutations. Third, the unusual phenotype of participant NF1-G6 is consistent with somatic mosaicism mainly confined to cells of neural crest origin (28). She had a mosaic NF1 phenotype, confirmed by molecular analysis: she presented with a glomus tumor, neurofibromas on the back and an intestinal ganglioneuroma but no freckling, learning disabilities, Lisch nodules or localized hyperpigmentation. Mosaicism in NF1 arises from a post-zygotic mutation of NF1 (41). In the case of NF1-G6, that mutation event likely occurred in the neural crest or a neural crest-derived cell, since both Schwann cells (neurofibroma) and intestinal ganglion cells (ganglioneuroma) are of neural crest origin. Accordingly, we hypothesize that glomus cells (glomus tumor) arise from myofibroblasts derived from neural crest stem cells.

In summary, we show that glomus tumors in NF1 arise secondary to bi-allelic inactivation of the tumor suppressor gene NF1 in $\alpha \mathrm{SMA}$-positive glomus cells. We observed that NF1inactivated glomus cells show increased MAPK signaling. Taken together, these data prove that glomus tumors of the fingers are an integral part of the tumor spectrum of NF1. We hope that an increased awareness of these tumors will improve their early diagnosis and treatment in individuals with NF1.

\section{Supplementary Material}

Refer to Web version on PubMed Central for supplementary material.

\section{Acknowledgements}

The authors thank Dena Hernandez and Andrew Singleton (National Institute on Aging of the U.S. National Institutes of Health) for their help with microarray processing, Julia Fekecs (NHGRI) for figure preparation and Les Biesecker (NHGRI) for discussions.

Grant Support

The work was supported in part by the Division of Intramural Research of the National Human Genome Research Institute (DRS) and the U.S. National Cancer Institute of the National Institutes of Health (DRS and Brigitte Widemann). Additional support was provided by the Institute for Promotion of Innovation through Science and Technology in Flanders (HB), research grants from the Fonds voor Wetenschappelijk Onderzoek Vlaanderen (G. 0578.06 and G.0551.08, EL), the Interuniversity Attraction Poles granted by the Federal Office for Scientific, Technical and Cultural Affairs, Belgium (IAP, 2007-2011; P5/25, EL), and by a Concerted Action Grant from the K.U.Leuven (EL). OM is a postdoctoral researcher with the Fund for Scientific Research Flanders (FWO-Vlaanderen).

\section{References}

1. Wallace MR, Marchuk DA, Andersen LB, et al. Type 1 neurofibromatosis gene: identification of a large transcript disrupted in three NF1 patients. Science 1990;249:181-6. [PubMed: 2134734]

2. Martin GA, Viskochil D, Bollag G, et al. The GAP-related domain of the neurofibromatosis type 1 gene product interacts with ras p21. Cell 1990;63:843-9. [PubMed: 2121370]

3. Knudson AG Jr. Hereditary cancer, oncogenes, and antioncogenes. Cancer Res 1985;45:1437-43. [PubMed: 2983882]

4. Brems H, Beert E, de Ravel T, Legius E. Mechanisms in the pathogenesis of malignant tumours in neurofibromatosis type 1. Lancet Oncol 2009;10:508-15. [PubMed: 19410195]

5. Rettig AC, Strickland JW. Glomus tumor of the digits. J Hand Surg [Am] 1977;2:261-5.

6. Strauchen JA. Germ-line mutations in nonsyndromic pheochromocytoma. N Engl J Med 2002;347:854-5. [PubMed: 12226162] author reply -5 .

7. McDermott EM, Weiss AP. Glomus tumors. J Hand Surg [Am] 2006;31:1397-400. 
8. Tsuneyoshi M, Enjoji M. Glomus tumor: a clinicopathologic and electron microscopic study. Cancer 1982;50:1601-7. [PubMed: 6288219]

9. Van Geertruyden J, Lorea P, Goldschmidt D, et al. Glomus tumours of the hand. A retrospective study of 51 cases. J Hand Surg [Br] 1996;21:257-60.

10. Klaber R. Morbus Recklinghausen with glomoid tumors. Proc Roy Soc Med 1938;31:347.

11. Sawada S, Honda M, Kamide R, Niimura M. Three cases of subungual glomus tumors with von Recklinghausen neurofibromatosis. J Am Acad Dermatol 1995;32:277-8. [PubMed: 7829715]

12. Okada O, Demitsu T, Manabe M, Yoneda K. A case of multiple subungual glomus tumors associated with neurofibromatosis type 1. J Dermatol 1999;26:535-7. [PubMed: 10487011]

13. Kim YC. An additional case of solitary subungual glomus tumor associated with neurofibromatosis 1. J Dermatol 2000;27:418-9. [PubMed: 10920593]

14. De Smet L, Sciot R, Legius E. Multifocal glomus tumours of the fingers in two patients with neurofibromatosis type 1. J Med Genet 2002;39:e45. [PubMed: 12161612]

15. Park YH, Choi SW, Cho BK, Houh W. Solitary type of glomus tumor developed in multiple sites. Ann Dermatol 1994;6:225-9.

16. Messiaen LM, Callens T, Mortier G, et al. Exhaustive mutation analysis of the NF1 gene allows identification of $95 \%$ of mutations and reveals a high frequency of unusual splicing defects. Hum Mutat 2000;15:541-55. [PubMed: 10862084]

17. Griffiths S, Thompson P, Frayling I, Upadhyaya M. Molecular diagnosis of neurofibromatosis type 1: 2 years experience. Fam Cancer 2007;6:21-34. [PubMed: 16944272]

18. Upadhyaya M, Kluwe L, Spurlock G, et al. Germline and somatic NF1 gene mutation spectrum in NF1-associated malignant peripheral nerve sheath tumors (MPNSTs). Hum Mutat 2008;29:74-82. [PubMed: 17960768]

19. Schouten JP, McElgunn CJ, Waaijer R, Zwijnenburg D, Diepvens F, Pals G. Relative quantification of 40 nucleic acid sequences by multiplex ligation-dependent probe amplification. Nucleic Acids Res 2002;30:e57. [PubMed: 12060695]

20. Correa, C Lopez; Brems, H.; Lazaro, C., et al. Molecular studies in 20 submicroscopic neurofibromatosis type 1 gene deletions. Hum Mutat 1999;14:387-93. [PubMed: 10533064]

21. Xu GF, Nelson L, O'Connell P, White R. An Alu polymorphism intragenic to the neurofibromatosis type 1 gene (NF1). Nucleic Acids Res 1991;19:3764. [PubMed: 1906608]

22. Lazaro C, Gaona A, Ravella A, et al. Novel alleles, hemizygosity and deletions at an Alu-repeat within the neurofibromatosis type 1 (NF1) gene. Hum Mol Genet 1993;2:725-30. [PubMed: 8353492]

23. Lazaro C, Gaona A, Xu G, Weiss R, Estivill X. A highly informative CA/GT repeat polymorphism in intron 38 of the human neurofibromatosis type 1 (NF1) gene. Hum Genet 1993;92:429-30. [PubMed: 8225327]

24. Lazaro C, Gaona A, Estivill X. Two CA/GT repeat polymorphisms in intron 27 of the human neurofibromatosis (NF1) gene. Hum Genet 1994;93:351-2. [PubMed: 8125490]

25. Kubota T, Nonoyama S, Tonoki H, et al. A new assay for the analysis of X-chromosome inactivation based on methylation-specific PCR. Hum Genet 1999;104:49-55. [PubMed: 10071192]

26. Uchida T, Ohashi H, Aoki E, et al. Clonality analysis by methylation-specific PCR for the human androgen-receptor gene (HUMARA-MSP). Leukemia 2000;14:207-12. [PubMed: 10637497]

27. Ng D, Thakker N, Corcoran CM, et al. Oculofaciocardiodental and Lenz microphthalmia syndromes result from distinct classes of mutations in BCOR. Nat Genet 2004;36:411-6. [PubMed: 15004558]

28. Maertens O, De Schepper S, Vandesompele J, et al. Molecular dissection of isolated disease features in mosaic neurofibromatosis type 1. Am J Hum Genet 2007;81:243-51. [PubMed: 17668375]

29. Maertens O, Prenen H, Debiec-Rychter M, et al. Molecular pathogenesis of multiple gastrointestinal stromal tumors in NF1 patients. Hum Mol Genet 2006;15:1015-23. [PubMed: 16461335]

30. Cichowski K, Santiago S, Jardim M, Johnson BW, Jacks T. Dynamic regulation of the Ras pathway via proteolysis of the NF1 tumor suppressor. Genes Dev 2003;17:449-54. [PubMed: 12600938]

31. Serra E, Puig S, Otero D, et al. Confirmation of a double-hit model for the NF1 gene in benign neurofibromas. Am J Hum Genet 1997;61:512-9. [PubMed: 9326316]

32. Emanuel PD. Juvenile myelomonocytic leukemia. Curr Hematol Rep 2004;3:203-9. [PubMed: 15087069] 
33. Yang MQ, Koehly LM, Elnitski LL. Comprehensive annotation of bidirectional promoters identifies co-regulation among breast and ovarian cancer genes. PLoS Comput Biol 2007;3:e72. [PubMed: 17447839]

34. Laios A, O’Toole SA, Flavin R, et al. An integrative model for recurrence in ovarian cancer. Mol Cancer 2008;7:8. [PubMed: 18211683]

35. Dahan O, Kupiec M. The Saccharomyces cerevisiae gene CDC40/PRP17 controls cell cycle progression through splicing of the ANC1 gene. Nucleic Acids Res 2004;32:2529-40. [PubMed: 15133121]

36. Steck E, Braun J, Pelttari K, Kadel S, Kalbacher H, Richter W. Chondrocyte secreted CRTAC1: a glycosylated extracellular matrix molecule of human articular cartilage. Matrix Biol 2007;26:30-41. [PubMed: 17074475]

37. Scholander PF, Van Dam L, Kanwisher JW, Hammel HT, Gordon MS. Supercooling and osmoregulation in arctic fish. J Cell Comp Physiol 1957;49:5-24.

38. Amir G, Rubinsky B, Basheer SY, et al. Improved viability and reduced apoptosis in sub-zero 21hour preservation of transplanted rat hearts using anti-freeze proteins. J Heart Lung Transplant 2005;24:1915-29. [PubMed: 16297800]

39. Cichowski K, Jacks T. NF1 tumor suppressor gene function: narrowing the GAP. Cell 2001;104:593604. [PubMed: 11239415]

40. Morrison SJ, White PM, Zock C, Anderson DJ. Prospective identification, isolation by flow cytometry, and in vivo self-renewal of multipotent mammalian neural crest stem cells. Cell 1999;96:737-49. [PubMed: 10089888]

41. Kehrer-Sawatzki H, Cooper DN. Mosaicism in sporadic neurofibromatosis type 1: variations on a theme common to other hereditary cancer syndromes? J Med Genet 2008;45:622-31. [PubMed: 18511569] 


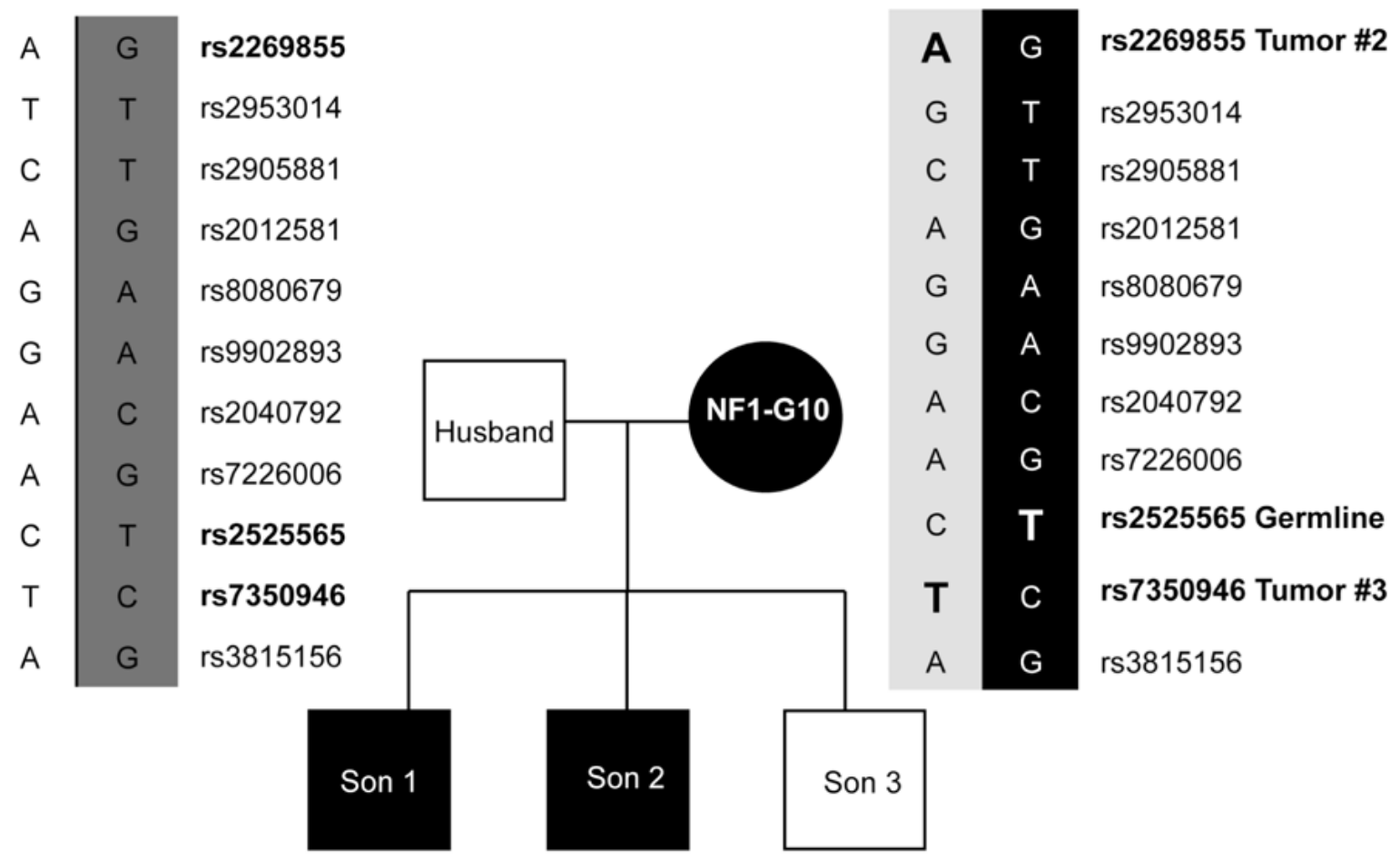

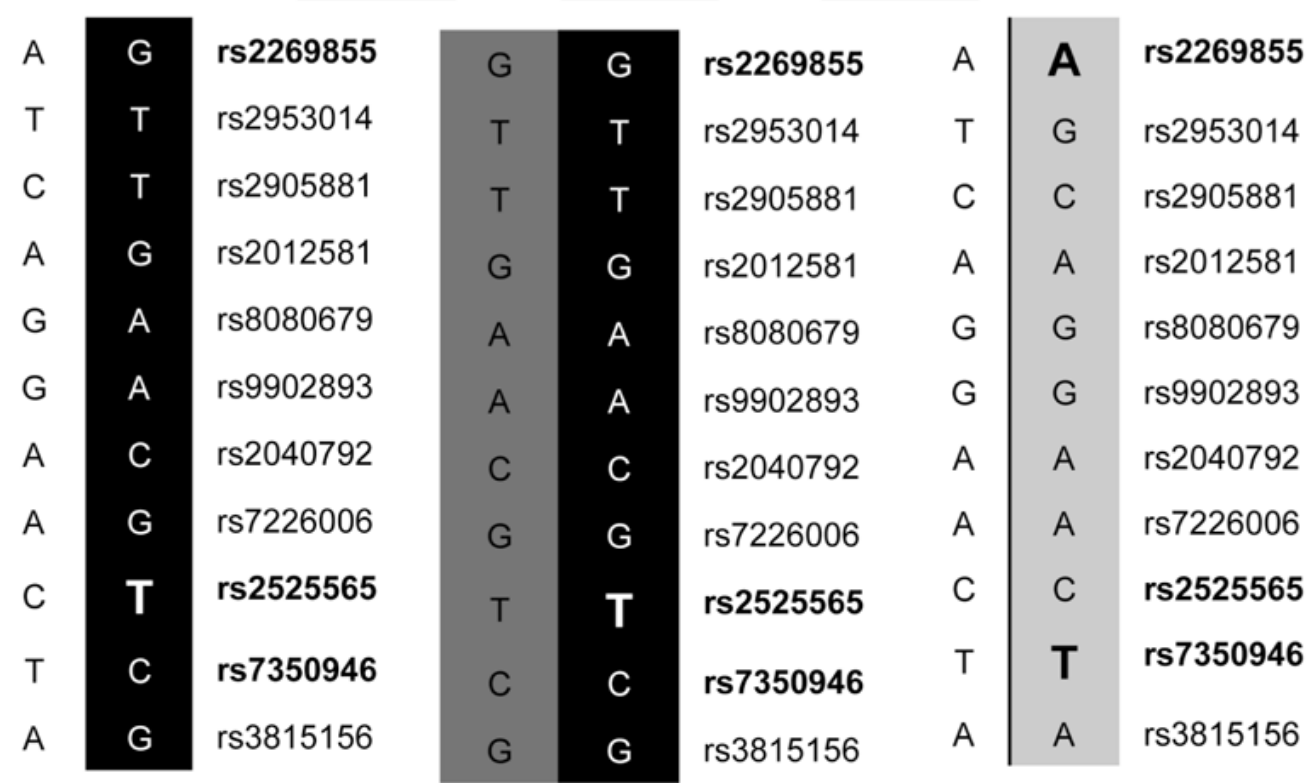

Figure 1.

Pedigree of NF1-G10 with phased 11 SNP NF1 haplotypes spanning $203 \mathrm{~kb}$ (5' end at top, 3 ' end at bottom). The wild-type haplotype harbors the two somatic NF1 mutations identified in her glomus tumors \#2 and \#3, evidence of $N F 1$ bi-allelic inactivation. SNP alleles sub-cloned with germline and somatic mutations are in bold. The maternal haplotype harboring the NF1 germline mutation (c.6789_6792 del TTAC, sub-cloned with SNP rs2525565, in black) cosegregates with the NF1 affectation status in the two affected sons (son 1 and son 2). The maternal haplotype harboring the two somatic NF1 mutations (light gray) is transmitted to the unaffected son (son 3). 
A

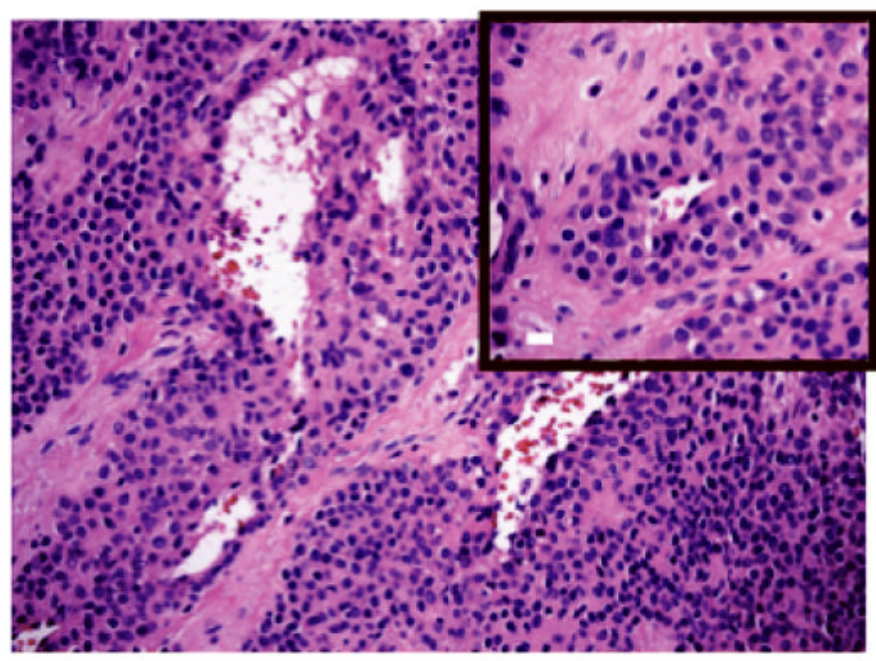

B

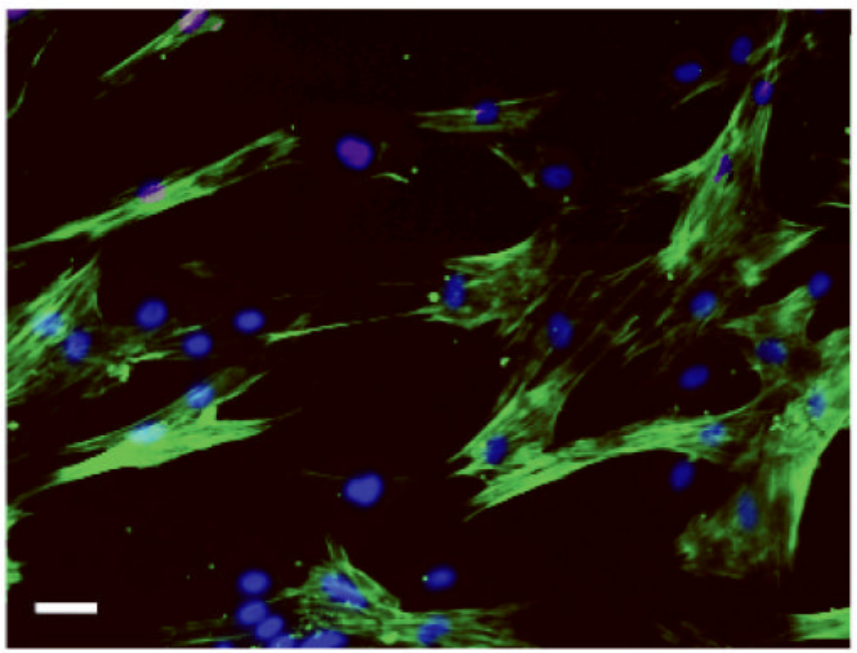

Figure 2.

A, photomicrograph of glomus tumor \#1 from NF1-G7 showing a uniform population of tumor cells with rounded nuclei and eosinophilic cytoplasm. Note the perivascular arrangement of the tumor cells (inset). Hematoxylin \& eosin stain, original magnification 250X, 400X (inset). Leica DMLB microscope. Bar $=15$ microns. $B$, immunocytochemistry of glomus tumorderived $\alpha$ SMA positive cells from tumor \#3 of NF1-G3. Nuclei are stained with DAPI (blue). $\alpha$ SMA-positive structures are green. Zeiss Axiophot fluorescent microscope. Bar $=15$ microns. 
A

NF1 tumor glomus NF1 tumor FB $600 \quad 515304560055153045$

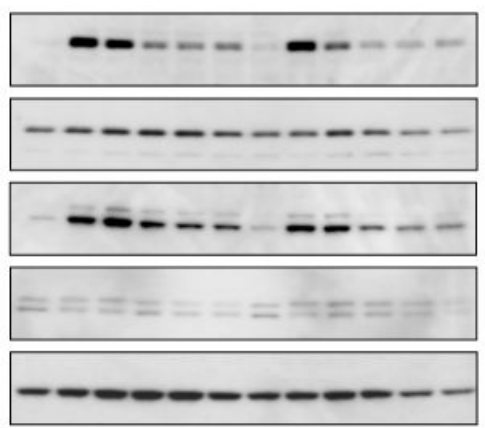

B

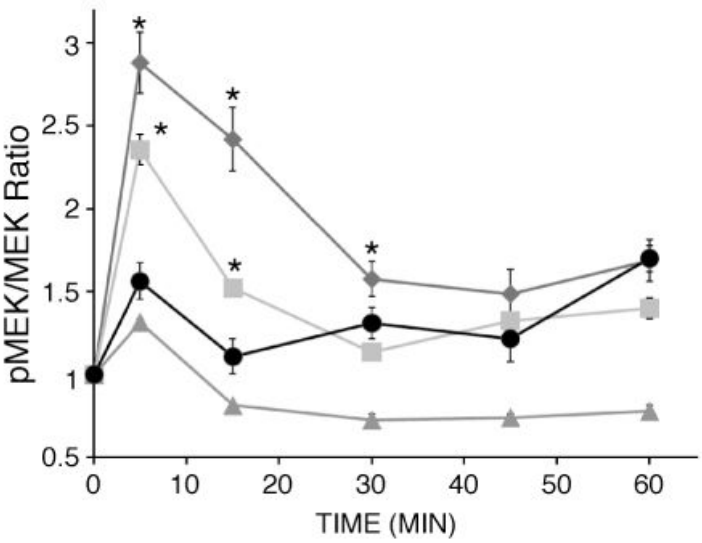

sporadic glomus control skin FB

$0 \quad 51530456005515304560$ TIME (MIN)

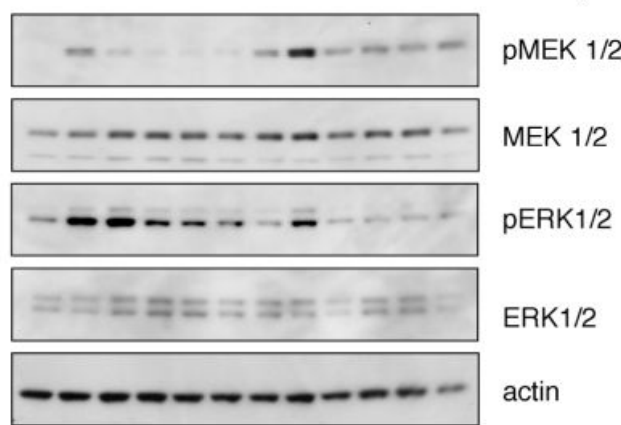

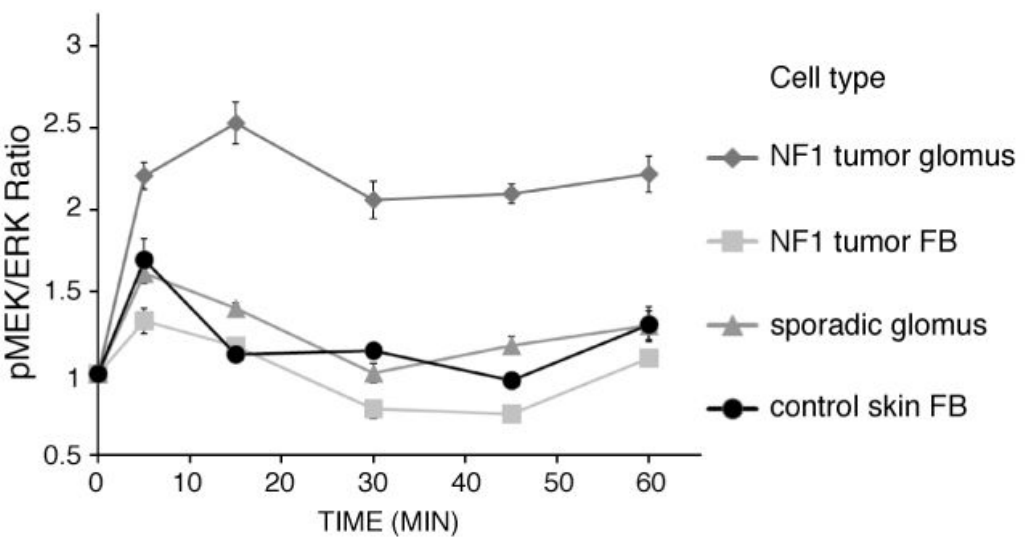

Figure 3.

aFGF stimulation of the RAS-MAPK pathway in cultured cells. Comparison of NF1-associated glomus tumor-derived glomus cells, NF1-associated glomus tumor-derived fibroblasts, sporadic glomus tumor-derived glomus cells and control skin fibroblasts before and at different time points after stimulation with aFGF. $A$, cell extracts immunoblotted with the indicated antibodies. $B$, ratios of $\mathrm{pMEK} / \mathrm{MEK}$ and $\mathrm{pERK} / \mathrm{ERK}$. All ratios were normalized to the ratio of the specific cell type before stimulation. Error bars represent standard deviation (SD). Average $\mathrm{pMEK} / \mathrm{MEK}$ and $\mathrm{pERK} / \mathrm{ERK}$ ratios of the four cell types were significantly different $\left(P=<10^{-3}\right)$. *Significant at the $5 \%$ level. 
A

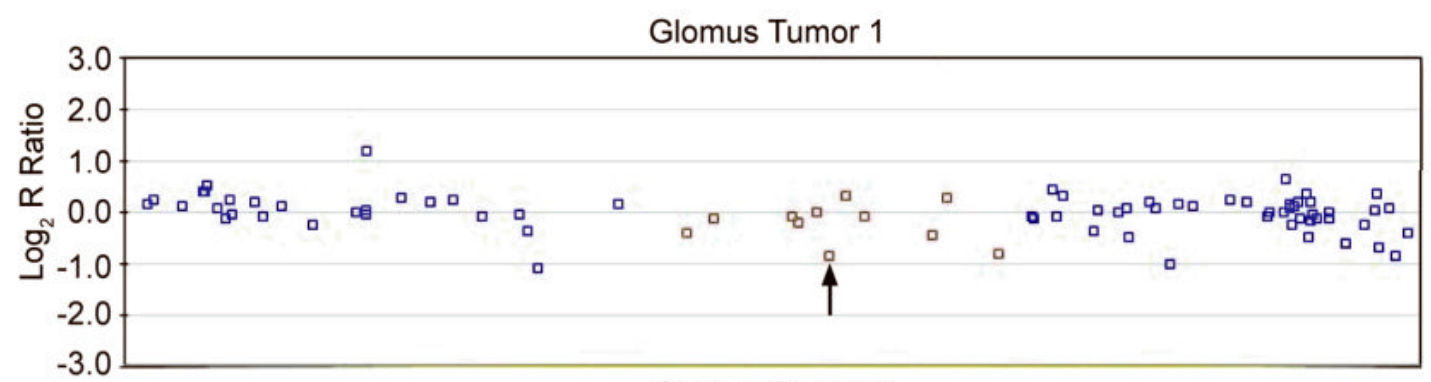

B

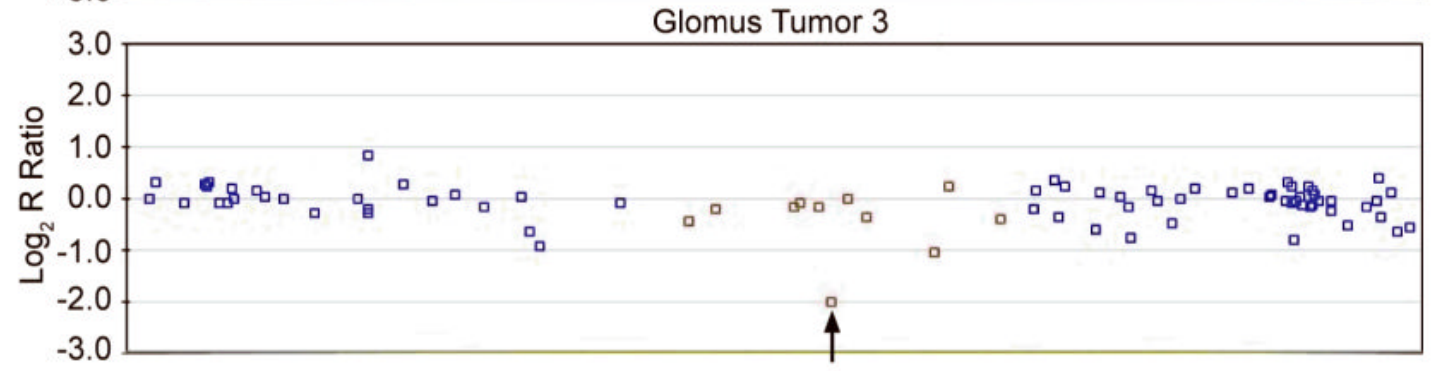

C

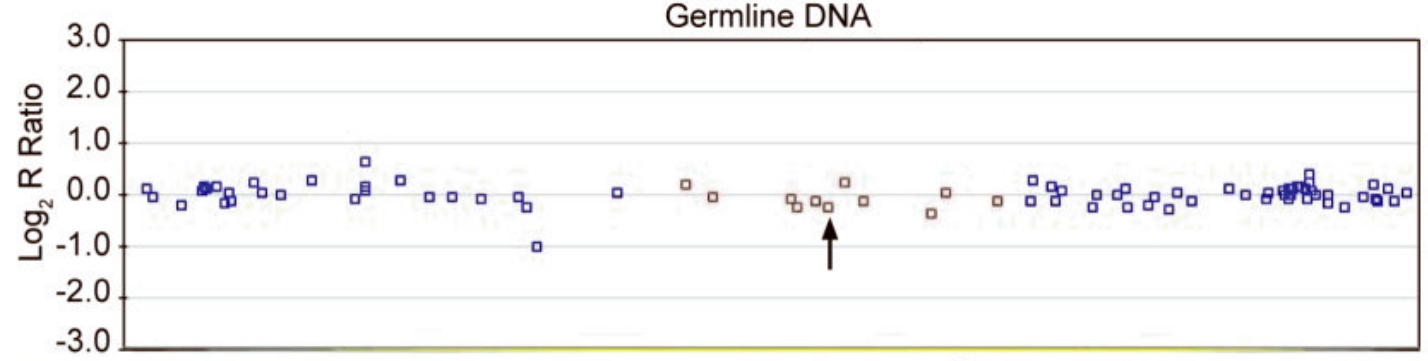

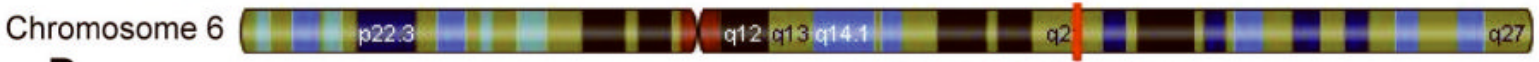

D

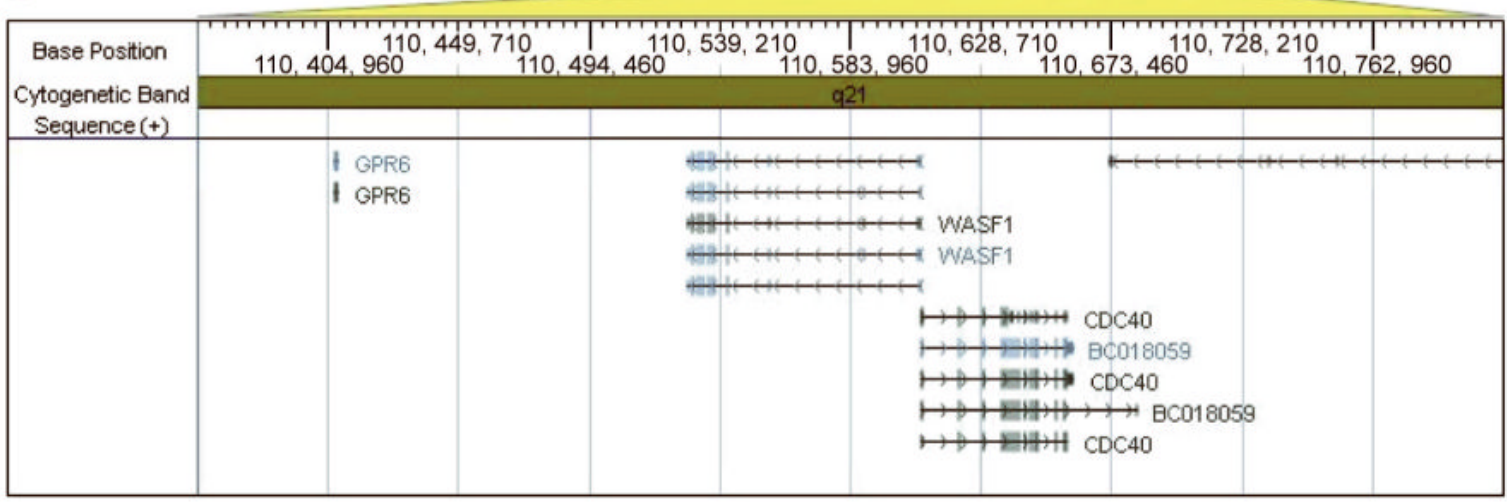

Figure 4.

Comparison of $\log _{2} \mathrm{R}$ ratio of SNP rs 4945851 (WASF1) and nearby SNP loci in glomus tumors $\# 1\left(\log _{2} \mathrm{R}=-0.9\right)$ and \#3 $\left(\log _{2} \mathrm{R}=-2.0\right)$ and germline DNA $\left(\log _{2} \mathrm{R}=-0.3\right.$, normal) from NF1G10. A $\sim 0.45 \mathrm{Mb}$ region $(110,360,210-110,807,710 \mathrm{bp}$ ) surrounding rs4945851 (arrow; position $110,603,926 \mathrm{bp}$ ) harboring 82 SNPs on chromosome 6 is shown in panels A-C (glomus tumor \#1, glomus tumor \#3 and germline sample). Locus rs4945851 and 10 adjacent SNPs (5 upstream and 5 downstream) are shown in red. The vertical axis is the $\log _{2} \mathrm{R}$ ratio of the intensity of the SNP-associated fluors. Panel D shows the genomic position in increments of 4,475 basepairs, cytoband (6q21) and surrounding genes. 


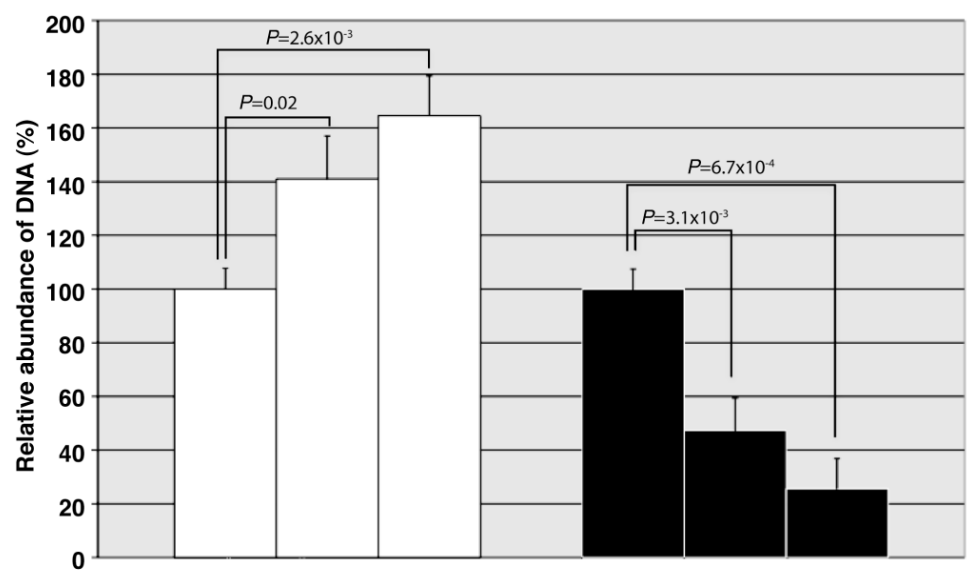

Figure 5.

Quantitative PCR of CRTAC1 and WASF1 loci in germline DNA (A), glomus tumor \#1 (B), and glomus tumor \#3 (C) from NF1-G10. Abundance of DNA at each locus was normalized to the quantity of DNA in the germline sample. 


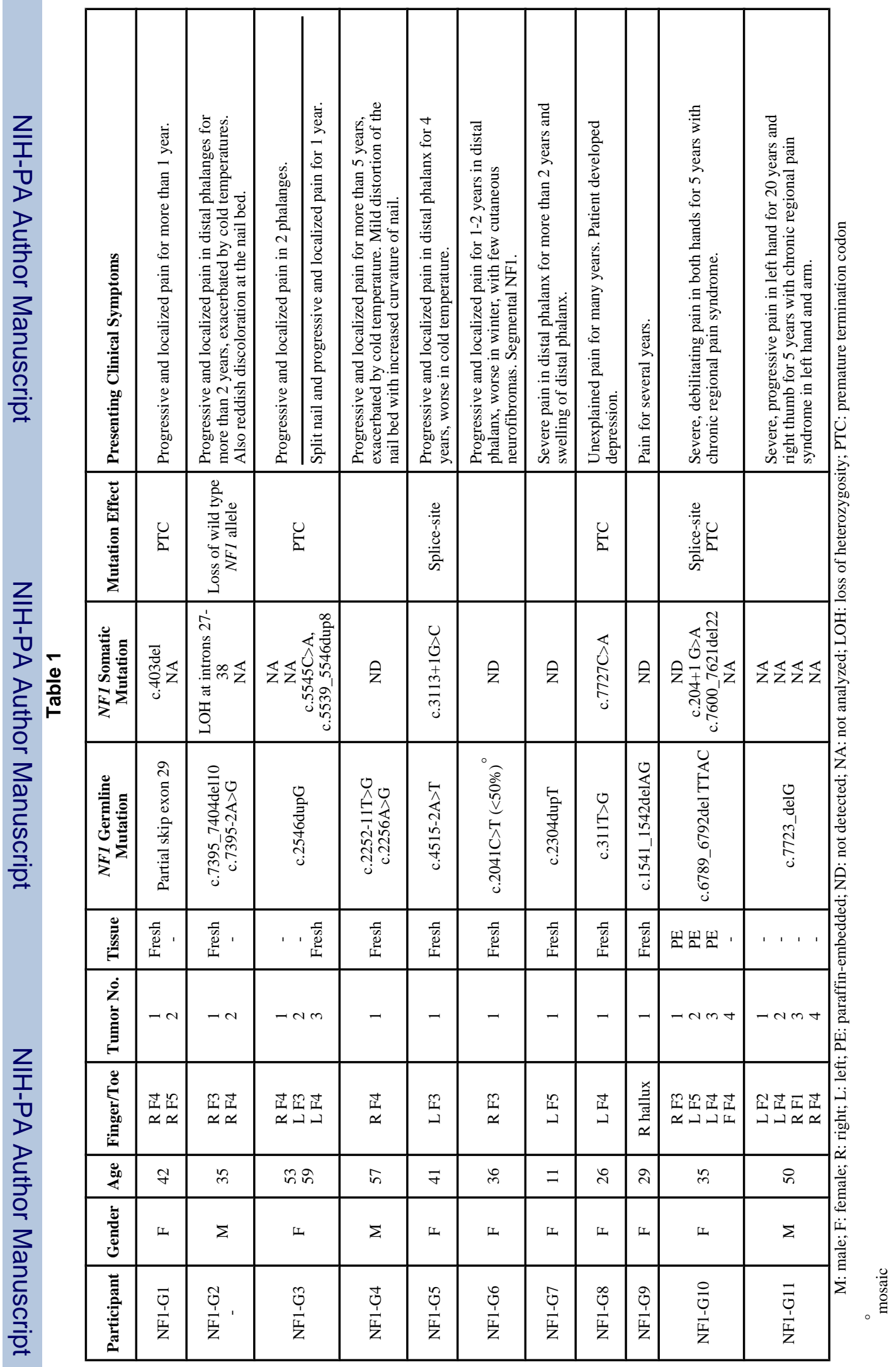

